\title{
Resonant second harmonic generation in random dielectric structures
}

\section{Marco Centini \\ Didier Felbacq \\ Diederick S. Wiersma}

Concita Sibilia

Michael Scalora

Mario Bertolotti

\author{
Dipartimento di Energetica , Università “La Sapienza" di Roma,Via Scarpa 16, 00161 Rome, Italy \\ Groupe d'Etude des Semi-Conducteurs UMR 5650 Bat. 21, CC074 Place Bataillon, 34095 Montpellier \\ Cedex 05, France \\ European Laboratory for Non-linear Spectroscopy (LENS) and INFM-Matis, Polo Scientifico, Via nello \\ Carrara 150019 Sesto-Fiorentino (Florence)Italy \\ Dipartimento di Energetica , Università “La Sapienza” di Roma,Via Scarpa 16, 00161 Rome, Italy \\ Charles M. Bowden Research Center, AMSMI-RD-WS-ST RDECOM, Redstone Arsenal, Bldg 7804, Al- \\ abama 35898-5000 \\ Dipartimento di Energetica , Università “La Sapienza” di Roma,Via Scarpa 16, 00161 Rome, Italy
}

We show that resonant second harmonic generation can be obtained in random dielectric structures. The scheme is based on internal resonances due to the optical counterpart of Anderson localization. By making use of different localization lengths at the fundamental and at the second harmonic frequencies, we predict a conversion efficiency that is four orders of magnitude higher than a bulk material and even one order of magnitude higher than an ideal phase matched slab of the same size. The method is highly insensitive to fabrication tolerances, and provides excellent angle tunability. [DOI: 10.2971/jeos.2006.06021]

Keywords: Nonlinear, random structures

\section{Introd uction}

Nonlinear properties of micro- and nano- structured materials are currently under intense investigation because of their ability to enhance the effective nonlinear response of a medium. Particular attention has been devoted to the study of second harmonic (SH) generation in photonic crystals (PC) [1]-[3]. Inside periodic structures, the nonlinear interaction between the fundamental (FF) and the $\mathrm{SH}$ fields is a phase sensitive process, governed by the fulfillment of the so called quasi phase matching (QPM) condition: $\mathbf{k}_{2 \omega}=2 \mathbf{k}_{\omega}+\mathbf{G}$, where $\mathbf{G}$ is the reciprocal lattice vector. These conditions are easier to achieve with respect to phase matching conditions in bulk media, thus allowing access to the high nonlinearity of mismatched semiconductors such as GaAs [4]. Finite size PCs also exhibit interesting properties due to cavity effects that become more intense closer to the band edge of the photonic structure, where the enhancement of $\mathrm{SH}$ generation has been theoretically predicted and experimentally verified $[5,6]$.

For particular geometries, the effect of field's localization in finite structure can be much more relevant than the fulfillment of QPM conditions [7]. In regimes where QPM is not crucial, one can obtain high efficiency by properly tailoring the structure in order to have high-field localization and good overlap between the FF and SH fields. Nevertheless, from a practical point of view, high enhancements factors are achievable if the structure is grown with a high degree of accuracy. This means that very precise control of layer thickness is re- quired, as reported in Ref. [8]. Even a small deviation from perfect periodicity causes line broadening of the resonances, loss of double resonant conditions, and decrease of field localization. The overall effect is a dramatic decrease of second harmonic production. In Ref. [8] a detailed study on the effect of weak disorder in one dimensional (1-D) photonic crystals is exploited to find conditions in which the disorder may be used to enhance the non linear process, rather than diminish it. Recently it has also been shown that random QPM can happen in bulk polycrystalline isotropic nonlinear materials [9]. Enhancement of second harmonic generation in strongly scattering porous $\mathrm{GaP}$ was experimentally investigated by V.A. Mel'nikov et al. in Ref. [10]. Their results indicate the importance of scattering effects in non-linear optical processes in strongly scattering media but the complexity of the system makes it difficult to have a deep insight on the phenomenon and a quantitative theoretical analysis.

A simpler and easier to understand scenario is presented if a structure with completely random layer thicknesses is considered. In this paper we show that, for 1-D systems, it is possible to find out the crucial parameters and the criteria to get high enhancement factors of SHG. Transmission spectra of randomly generated, finite size, multilayer stacks are characterized by the presence of randomly distributed transmission peaks. They originate from resonances created by localized modes inside the sample. This effect is the optical ana- 
logue of Anderson localization, originally studied for electrons in disordered conductors [11]. In 1-D systems, Anderson localization effects manifest themselves provided sample size is large compared to the localization length, which depends on frequency and the degree of disorder. Even in the presence of localized Anderson states, so-called "necklace" modes $[12,13]$ can also appear. They are characterized by almost unitary transmission, and are particular relevant to finite size structures. A necklace state is obtained when more than one resonance exists in the sample at very similar frequencies.

Here we show that it is possible to obtain highly efficient second harmonic generation in randomly patterned dielectric materials. Our results demonstrate that one can take advantage of a combination of strongly and weakly confined localized modes, as well as optical necklace states, for resonant second harmonic generation, even if the material is completely disordered. The converted SH energy is even an order of magnitude higher than that of the ideal case of a perfectly phasematched slab (etalon) of the same size.

\section{LINEAR P R O P E R TIES: L 0 - CALISED STATES AND NECK- LACE STATES}

We consider a structure composed of two different dielectric materials, with "unit" layer optical thickness having a mean value of $\lambda_{0} / 4$ (with $\lambda_{0}=1 \mu \mathrm{m}$ ) with $10 \%$ Gaussian fluctuations. The low index material (L) is assumed to be linear and non-dispersive. The high index material $(\mathrm{H})$ has linear chromatic dispersion and is also nonlinear. A stronger degree of disorder is introduced by randomly choosing the sequence of layers, assuming that each layer has $50 \%$ of probability to be $\mathrm{H}$ or L. Data for materials used in our calculation are the same as those of Ref.[14], namely $n_{L}=1.45$ and $n_{H}^{2}=4.9048+0.11768 /\left(\lambda^{2}-0.0475\right)-0.027169 \lambda^{2}$ dielectric constant of $\mathrm{LiNbO}_{3}$ ordinary wave).
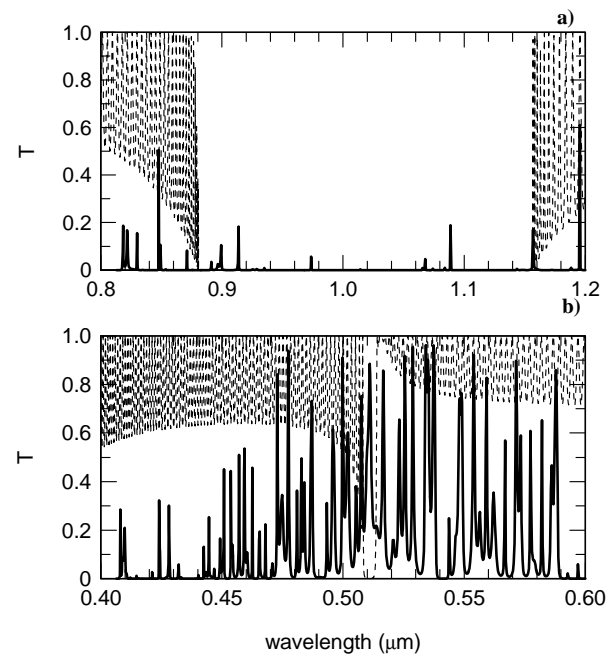

FIG. 1 Transmission spectra at normal incidence (wavelength ranges in the near infrared (a) and in the visible (b)) for a 250 layer periodic structure (thin dashed line) and for a random structure (thick line).
In Figures $1 \mathrm{a}$ and $1 \mathrm{~b}$, we show a typical transmission spectrum for a randomly generated structure, obtained by alternating 250 "unit" layers (thick line) for different wavelength regions. We compare this to the spectrum of a perfect 250-layer periodic structure (thin, dashed line), and note the following analogies: inside the gap, (Figure 1a) of the periodic structure, the random structure's spectrum exhibits an almost zero transmittance with the exception of a few, very narrow resonances. This appears to be the right tuning wavelength range for the pump field, because sharp resonances in the transmission spectrum are related to high field localization inside the sample. On the other hand, the features of transmission spectra in the $\mathrm{SH}$ wavelength range $(0.4$ to $0.6 \mu \mathrm{m})$ suggest a totally different, much more interesting and useful behavior, characterized by very closely spaced, high, and relatively broad transmission resonances (Figure 1b). We will later focus on the transmission properties of the $\mathrm{SH}$ wavelength range.
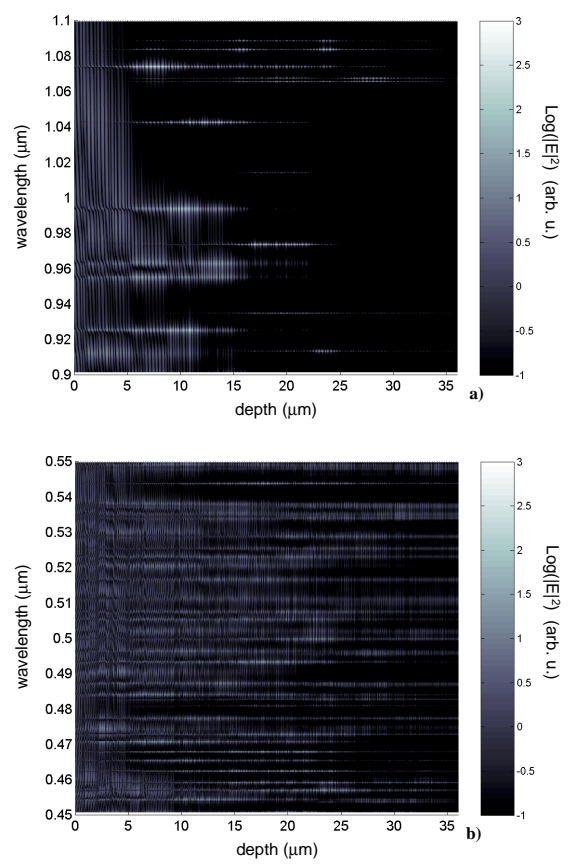

FIG. 2 a) FF field profile square modulus as a function of wavelength inside the structure. We note that both Anderson localized states and necklace states are present. b) $\mathrm{SH}$ field profile square modulus; there is a high density of weakly confined states available.

In Figures $2 \mathrm{a}$ and $2 \mathrm{~b}$ we plot the field intensity (logarithmic scale) as a function of the position inside the structure for the pump and $\mathrm{SH}$ wavelength ranges. We note that in the pump wavelength range there are several highly localized states (for example the state at approximately $\lambda=1.075 \mu \mathrm{m}$ ) where local intensities may be magnified with respect the input intensities up to three orders of magnitude. We also note the presence of necklace states (approximately at $\lambda=1.09 \mu \mathrm{m}$ for example) due to coupling of two or more resonant states at very close wavelengths. On the other hand, the $\mathrm{SH}$ wavelength range is characterized by high transmittance but weakly confined states. This different behavior of the FF and $\mathrm{SH}$ fields is due to the large difference in the respective localization lengths. We calculated the localization length at $\lambda_{0}=1 \mu \mathrm{m}$ and at $\lambda_{o} / 2$ by 
using the formula [13]:

$$
\langle\ln T\rangle=-\frac{L}{\xi}
$$

where $\mathrm{L}$ is the length of the sample, $\mathrm{T}$ is the modulus of the transmission coefficient, the brackets denote an average over many realizations of the disorder, and $\xi$ is the localization length. We numerically performed an average over 1000 different realizations. The calculated localization length at the FF is $\xi_{F F} \sim 7 \mu \mathrm{m}$, while at the $\mathrm{SH} \xi_{S H} \sim 37 \mu \mathrm{m}$. This means that the structure is optimized for maximum scattering at $\lambda_{0}=$ $1 \mu \mathrm{m}$, where the localization length has its minimum value and mode confinement is highest. In contrast, at $\lambda_{0} / 2, \xi_{S H}$ would diverge if we neglected material dispersion and the $10 \%$ Gaussian disorder in the layer thickness. From the point of view of localization length, at $\lambda_{0} / 2$ only the $10 \%$ Gaussian disorder introduced in layer thickness, matters. Therefore, the localization length in this range is much longer than in the FF wavelength regime. We note that it is actually of the same order of the total sample thickness (approx. 35.7 $\mu \mathrm{m}$ ), which is consistent with the observed absence of localized modes in this regime (Figure $2 b$ ).

\section{NON-LINEAR PROPERTIES: EN- HANCEMENT OF SECOND HAR- MONIC GENERATION}

In order to investigate $\mathrm{SH}$ generation we extended the model presented in Ref. [5] to the case of an arbitrary choice of the incidence angle of the pump in the plane of incidence $x-z$ perpendicular to the layers' interface planes. For a given monochromatic plane wave characterized by an angular frequency $\omega$ and a value of the x-component of the wavevector $\left(k^{x}\right)$ the field is described as a superposition of left to right (LTR) and right to left (RTL) linear solution of Helmoltz equations normalised with respect to a unitary input field with complex envelope functions $A_{n}^{+}(z)$ and $A_{n}^{-}(z)$ as:

$$
\begin{aligned}
\mathbf{E}_{n}(z, x)=A_{n}^{+}(z) & \boldsymbol{\Phi}_{n}^{+}(z) \exp \left(i k_{n}^{x} x\right) \\
& +A_{n}^{-}(z) \boldsymbol{\Phi}_{n}^{-}(z) \exp \left(i k_{n}^{x} x\right)
\end{aligned}
$$

where $\mathrm{n}=\mathrm{FF}, \mathrm{SH}$; and $\boldsymbol{\Phi}_{n}^{ \pm}(z)$ can be calculated using standard matrix transfer techniques by applying the proper boundary conditions to all field's components. We note that for plane waves the complex envelope functions $A_{n}^{+}(z)$ and $A_{n}^{-}(z)$ can only be function of the $\mathrm{z}$ variable in order to keep the proper wave front plane. Following the multiple scale expansion procedure shown in [5] with the vectorial fields expressed as in Eq.(2) and focusing our attention to the process of collinear second harmonic generation from a forward propagating undepleted pump, we arrive to a formula for the envelopes of the $\mathrm{SH}$ generated field in forward $A^{+}(L)$ and backward $A^{-}(0)$ directions:

$$
A_{S H}^{+}(L)=\frac{i \omega L\left(A_{F F}^{+}(0)\right)^{2}\left[\Gamma_{(S H,+)}^{(+,+)} p_{S H}^{(-,-)}-\Gamma_{(S H,-)}^{(+,+)} p_{S H}^{(+,-)}\right]}{c\left[p_{S H}^{(+,+)} p_{S H}^{(-,-)}-p_{S H}^{(+,-)} p_{S H}^{(-,+)}\right]} ;
$$

$A_{S H}^{-}(L)=\frac{-i \omega L\left(A_{F F}^{+}(0)\right)^{2}\left[\Gamma_{(S H,-)}^{(+,+)} p_{S H}^{(+,+)}-\Gamma_{(S H,+)}^{(+,+)} p_{S H}^{(-,+)}\right]}{c\left[p_{S H}^{(+,+)} p_{S H}^{(-,-)}-p_{S H}^{(+,-)} p_{S H}^{(-,+)}\right]} ;$

where $z=0$ znd $z=L$ are the coordinates of the first and the last interface ( being $L$ the total length of our structure) and :

$$
p_{S H}^{(k, l)}=\frac{-i c}{2 \omega_{S H} L} \int_{0}^{L}\left(\boldsymbol{\Phi}_{S H}^{k^{*}} \cdot \frac{d}{d z} \boldsymbol{\Phi}_{S H}^{l}\right) d z ; \quad \text { for } \mathrm{k}, \mathrm{l}=+,-
$$

and

$$
\Gamma_{(S H, j)}^{(k, l)}=\frac{i}{L} \int_{0}^{L}\left(\boldsymbol{\Phi}_{S H}^{j^{*}} \cdot \hat{d}^{(2)}: \boldsymbol{\Phi}_{F F}^{k} \cdot \boldsymbol{\Phi}_{F F}^{l}\right) d z ; \quad \text { for } \mathrm{k}, \mathrm{l}, \mathbf{j}=+,-.
$$

Sustituting Eqs.(3a,3b) into Eq.(2) it is possible to calculate the outgoing $\mathrm{SH}$ field. Assuming a tunable CW pump, and sweeping the wavelength range of interest, we calculate the spectrum of the generated second harmonic signal. We depict the spectrum of the generated $\mathrm{SH}$ energy as a function of pump wavelength in Figure 3 (thin solid line).

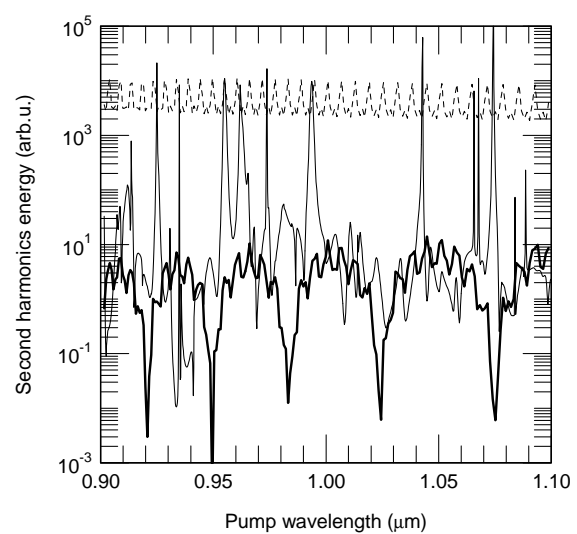

FIC. 3 Second harmonic energy vs. pump's wavelength generated by our sample (solid thin line) compared to the generated second harmonic from an etalon made of the same material and of the same length (solid thick line) and the same etalon for an ideal perfect phase-matched material.

We note that there are high peaks of $\mathrm{SH}$ generation when the pump is tuned at a wavelength corresponding to a localized Anderson mode, and the $\mathrm{SH}$ is tuned to a non-localized high transmittance mode. Indeed, these modes are efficiently coupled with the output of the structure and the generated second harmonic signal can easily exit the structure. At the same time, the pump is strongly localized inside a portion of the structure, where the nonlinear process is enhanced. The high transmission states available to the $\mathrm{SH}$ field make it possible to have a high probability for a given Anderson pump state to transfer energy to the second harmonic mode. Upon increasing the system size, Anderson modes occur with increasing internal energy density and decreasing bandwidth. This means that upon increasing the system size the production of second harmonic generation increases. However, the chance of obtaining overlap between the fundamental and second harmonic frequency decreases. 
Finally, we compared the efficiency of our structure with the unpatterned, nonlinear slab of the same thickness, and with an ideal perfectly phase matched bulk. In both cases we considered the interference effect due to multiple reflections resulting from the refractive index discontinuity at the input (air $\left./ n_{H}\right)$ and output $\left(n_{H} /\right.$ air) parallel interfaces. We note that the $\mathrm{SH}$ production in the non phase matched single slab (solid thick line) is strongly affected by the phase mismatch which would give almost zero signal when the slab length is equal to an even number of coherence lengths. Fast oscillations are due to superposition of the modulation of the linear transmittance at pump and SH wavelengths. Out of resonance, local phase matching conditions govern the nonlinear dynamics also for the random structure (solid thin line). As expected, we find that the average $\mathrm{SH}$ signal generated by our structure over the given wavelength range is of the same order as the signal of an unmatched slab. Nevertheless, we note that, for some wavelengths, the $\mathrm{SH}$ generation may be enhanced by 3-4 orders of magnitude with respect to its average value. Finally, we observe enhancements of SH production of over an order of magnitude with respect to an ideal, perfectly phase matched etalon made of the same nonlinear material of the same length as the structure (dashed line). Due to the small size of our structures, overall conversion efficiencies might be not as high as in long phase matched birefringent crystals or QPM waveguides. Nevertheless the structures we propose have a high efficiency versus size ratio and are interesting for applications where miniaturized and integrated devices are required.

Resonant conditions for SH generation can generally be found easily if the degree of disorder and the total thickness of the structure are properly tailored. Obviously, different realizations, or "codes", produce different spectra; Nevertheless the fact that localization lengths at FF and $\mathrm{SH}$ regimes are different is a statistical property as shown in Eq.(1). Thus the spectral behavior in terms of probability to find an Anderson localized state for the pump and an allowed non localized state for second harmonic are the same. The rule of thumb is to calculate the localization lengths at FF and $\mathrm{SH}$ by averaging over many realizations of the disorder and choose the total length of the structure so that localization length at the FF field is shorter than the structure (Anderson localized states appear) but localization length at $\mathrm{SH}$ is longer or comparable to the structure size in order to have high density of allowed, non localized, high transmittance states. For every realizations enhanced SH generation will be found at different wavelengths with respect to the ones showed in the example. This is usually not a problem for applications where a tunable pump is considered. If we seek SH generation at a specific wavelength, some efforts are required in order to optimize the device.

Moreover, the high density of allowed states that display unitary transmission in the $\mathrm{SH}$ wavelength range makes it possible to change the incidence angle of the pump in order to tune it at a desired, highly localized state, thus maintaining good resonant $\mathrm{SH}$ generation, as shown in Figure 4.

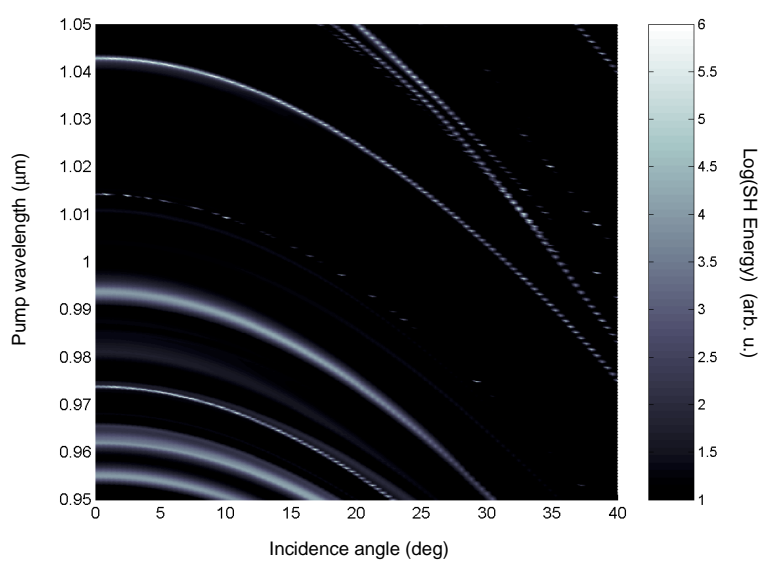

FIG. 4 Total second harmonic generated energy as a function of pump wavelength and incidence angle. We note high angle tunability of the pump.

Changing the pump incidence angle from 0 to 40 degrees causes the second harmonic peaks to blueshift, keeping high values of enhancement with respect to the background level. Switching between different modes, we have a tunability range of more than $100 \mathrm{~nm}$. This remarkable property adds flexibility in terms of tunability and fabrication sensitivity to this new kind of devices.

\section{CONCLUSIONS}

In conclusion, we have proposed a new method to achieve high enhancements of second harmonic generation using random layered structured. This method does not rely on phase matching, is highly insensitive to fabrication tolerances, and it provides excellent tunability in both incident angle and wavelength. It is based on the existence of both highly confined Anderson localized modes and weakly confined modes in finite random structures. The efficiency is enhanced by more than 4 orders of magnitude with respect to nonlinear bulk material of the same size, and by more than one order of magnitude compared to what can be obtained from an ideally phase matched bulk material. The authors wish to acknowledge the NoE "Phoremost" and the CNR-CNRS agreement for partial financial support.

\section{References}

[1] M. Bertolotti, C.M. Bowden and C. Sibilia, AIP vol. 560 (AIP, Melville 2001)

[2] Topics in Applied Physics, V.M. Shalaev, ed., Vol. 82 (SpringerVerlag Berlin Heidelberg 2002)

[3] E. Centeno, Opt. Lett. 30, 1054 (2005)

[4] M. Scalora, M. J. Bloemer, C.M. Bowden, G. D’Aguanno et al.: April 2001 Optics at Photonics News 39

[5] G. D’Aguanno, M. Centini, M. Scalora, C. Sibilia et al. J. Opt. Soc. Am. B 19, 2111 (2002)

[6] Y. Dumeige, I. Sagnes, P. Monnier, P. Vidakovic et al. Phys. Rev Lett. 89, 043901 (2002) 
[7] M. Centini, G. D’Aguanno, L. Sciscione, C. Sibilia et al. Opt. Lett. 29, 1924 (2004)

[8] D.Faccio, F.Bragheri, Phys. Rev. E, 71, 057602 (2005)

[9] M. Baudrier-Raybaut, R Haidar, Ph. Kupecek, Ph. Lemasson, E. Rosencher, Nature 432, 374 (2004)

[10] V.A. Melnikov, L.A. Golovan, S. O. Konorov D.A. Muzychenko et al,
Appl. Phys. B 79, 225 (2004)

[11] P. W. Anderson, Phys. Rev. 109, 1492 (1958)

[12] J. B. Pendry, J. Phys. C 20, 733 (1987)

[13] J. Bertolotti, S. Gottardo, D. S. Wiersma, M. Ghulinyan, L. Pavesi, Phys. Rev. Lett. 94, 113903, (2005)

[14] T. Ochiai, K. Sakoda, Optics Express 13, 9094 (2005) 